\title{
Google Classroom testimonial en la gestión de proyectos de emprendimiento
}

\section{Google Classroom testimonial in the management of entrepreneurship projects}

\author{
Jacqueline Rupay de la Sota*I y Doris Esther Castillo Villegas*I \\ *Departamento Académico de Educación, Facultad de Educación, Universidad Nacional del Centro del Perú (UNCP), Huancayo-Perú.
}

\section{Resumen}

Google Classroom testimonial es una alternativa de innovación para la educación en estos tiempos, actualmente la tecnología ofrece una fácil comunicación e interacción de docentes y estudiantes en el aprendizaje que permite compartir información, a la vez enviar y recibir trabajos. Por otro lado, se empleó la metodología de la investigación cualitativa fenómeno educativa. Cuyos resultados ayudaron al trabajo pedagógico de enseñanza aprendizaje para la gestión de proyectos de emprendimiento, además promueve en los estudiantes adaptarse a diversos cambios, como el trabajo en equipo, asumir responsabilidades individuales y colectivas, tener ideas innovadoras, asumir riesgos y finalmente lograr objetivos propuestos.

Palabras Claves: Google Classroom, herramienta tecnológica, proyectos de emprendimiento.

\begin{abstract}
Google Classroom testimonial is an innovative alternative for education in these times, currently technology offers easy communication and interaction of teachers and students in learning that allows information to be shared, while sending and receiving jobs. On the other hand, the methodology of qualitative phenomenal educational research was used. Whose results helped the pedagogical work of teaching-learning for the management of entrepreneurship projects, in addition, it promotes in students adapting to various changes, such as teamwork, assuming individual and collective responsibilities, having innovative ideas, taking risks and finally achieving proposed objectives.
\end{abstract}

Keywords: Google Classroom, technological tool, entrepreneurship projects.

1 Correspondiencia: Jacqueline Rupay de la Sota y Doris Esther Castillo Villegas, jaely347@gmail.com / doriscas0812@gmail.com 


\title{
Depoimento do Google Classroom na gestão de projetos de empreendedorismo
}

\begin{abstract}
Resumo
O Google Classroom é uma alternativa inovadora para a educação nos tempos atuais, atualmente a tecnologia oferece fácil comunicação e interação de professores e alunos na aprendizagem que permite o compartilhamento de informações, no envio e recebimento de empregos. Por outro lado, foi utilizada a metodologia da pesquisa qualitativa fenomenal-educacional. Cujos resultados auxiliaram o trabalho pedagógico de ensinoaprendizagem para a gestão de projetos de empreendedorismo, além disso, promove nos alunos a adaptação às diversas mudanças, como o trabalho em equipe, assumindo responsabilidades individuais e coletivas, tendo ideias inovadoras, assumindo riscos e finalmente atingindo os objetivos propostos.
\end{abstract}

Palavras Claves: Google Classroom, ferramenta tecnológica, projetos de empreendimento.

\section{Classroom Gooblem sullull kan gestión proyectos chay emprendimientum nisqampi}

\section{Uchuycha}

Geoogle Classroom sullull kan musuqyachanayachaqkupaq kaypunchaupaq. Kaytecnologiamsasachakunrimananchiqpaq amautakunawanyachaqkunawanyachaptin, rimanakunapaqchaymanta apanqa, llamkaykunatachasquisun. Kayruwana tecnológicakallpanchanqa de proyecto de emprendimiento nisqanyachaskunamchaytaruanqaallin llamkaykananpaqllapallanrunakunapaq, allinallin yuyayninwan, sumaqatiqtukukuykanampaq.

Hatun apup simi: Google Classroom, llamkana tecnológica allin proyectokuna. 


\section{Introducción}

Google Classroom testimonial, es una metodología de la investigación cualitativa fenómeno educativa. Conformado por un registro testimonial, propuesta de movilización, solidaridad sincrónica, grupo o movimiento al que pertenece, veracidad de los eventos, objetividad y solidaridad, producción circulación y recepción, apreciación de las realidades, descripción del testimonio, interpretación de la verdad emergente. Tiene por finalidad conocer una realidad social generalmente inhumana por medio del testimonio de alguno de sus protagonistas o testigos directos.

La narrativa testimonial, se soporta en la interacción de la vida como el todo y la vivencia como la parte, en tanto que ambos necesariamente se complementan. Lo vivido es parte de nosotros y forma el sentido significado al que pertenece concienciarte. Describe la forma natural en que los protagonistas relatan el testimonio de las vivencias y contenidos, para la movilización respectiva del cambio de las prácticas opresoras. Interpreta la narrativa de urgencia, pretende el destierro de prácticas inhumanas, enlazando la historia, la memoria y el testimonio del autor ligado directamente con los movimientos y cambios sociales. (Cerrón Rojas, 2019).

Google Classroom permite ahorrar tiempo, organizar las clases y comunicarse con los alumnos. Ahora, la aplicación no tiene las mismas funciones de otras plataformas como Blackboard, pero es más intuitivo para utilizar, gratuito, y la aplicación puede bajarse a cualquier dispositivo móvil como el celular. Por otro lado, también resulta más atractivo para los estudiantes, ya que su plataforma se asemeja a una red social, como Facebook, con un muro o tablero, donde aparecen las asignaciones, comentarios del profesor y estudiantes, fechas importantes, anuncios tanto de los estudiantes como del profesor. Por tanto, Google Classroom es realmente una aplicación de enseñanza enfocada en la interacción social. (Velez, 2016).

Los entornos virtuales de aprendizaje (EVA) ofrecen el desarrollo de nuevos contextos para el aprendizaje, flexibilizan el espacio educativo otorgando diversidad, rompen el esquema de espacio tiempo promoviendo trabajo colaborativo, siendo este un elemento dominante en el proceso de enseñanza-aprendizaje. (Rodríguez, 2019)

Gestiona proyectos de emprendimiento económico o social, es cuando el estudiante lleva a la acción una idea creativa movilizando con eficiencia y eficacia los recursos, tareas y técnicas necesarias para alcanzar objetivos y metas individuales o colectivas con la finalidad de resolver una necesidad no satisfecha o un problema económico, social o ambiental. (MINEDU, 2016)

Las categorías que estable la narrativa testimonial en la metodología fenómeno educativa constituye:

El registro testimonial, es un reconocimiento de una determinada situación que se considera de relevancia. Constituye el uso de un leguaje formal, fluidez de vocabulario, oraciones completas y uso de sinónimos y pronombres. Tiene por finalidad de dar a conocer experiencias vivenciales a otras personas

Se realizó un conversatorio virtual con estudiantes de 5to. Grado de educación secundaria de la provincia de Yauli, distrito La Oroya y departamento de Junín, teniendo una duración de 38 minutos, utilizando la plataforma Zoom. El cual fue de gran ayuda para conocer las experiencias de los estudiantes.

La propuesta de movilización, es una oferta o invitación que alguien dirige a otro o a otros, persiguiendo algún fin. Permite concretar una idea, una relación en el aprendizaje. Con la intención de compartir el aprendizaje y beneficios del Google Classroom sobre gestión proyectos de emprendimiento. Realizado en el trabajo remoto de "Aprendo en casa".

Los estudiantes afirmaron que les fue de gran ayuda para asistir en diferentes clases, verificar los documentos en word, power point y excel, videos, imágenes, revisar y enviar las tareas. Asimismo, para recibir información acerca de las calificaciones en tiempo real. Y por último docentes y estudiantes interactuaron activamente.

En cuanto a la solidaridad sincrónica, es una solidaridad intra-grupal. Consiste el desarrollo autóctono en base a los patrones de la cultura de cada grupo social. Debe permitir a los distintos pueblos del planeta establecer y 
ajustar sus políticas de desarrollo teniendo en cuenta los intereses de la colectividad mundial. Implica aunar en un mismo tiempo histórico las necesidades y prioridades de acción sobre el medio.

El MINEDU establece el uso de recursos tecnológicos para el desarrollo de Aprendo en Casa, el docente asumió el reto de aplicar Google Classroom para el logro de desarrollo en la gestión de proyectos de emprendimiento. Considerando las necesidades y/o problemas económicos o sociales de la realidad de los estudiantes.

En estos tiempos de pandemia, los estudiantes a través de sesiones de aprendizaje utilizando recursos tecnológicos, y bajo la guía y orientación de docentes, adquieren conocimientos, habilidades, actitudes. Para lograr una formación integral.

Los padres de familia son de gran apoyo en la educación de sus menores hijos, brindándole un celular, laptop, el pago del internet para acceder a esta plataforma. Y lograr el objetivo final aprendizaje significativo del estudiante.

Finalmente, la plana directiva de las instituciones educativas ayudó con las capacitaciones, en cuanto al uso de las herramientas digitales para contribuir en el aprendizaje de docentes y estudiantes, dichas acciones permitieron continuar con el aprendizaje a distancia.

En el grupo un movimiento al que pertenece es femenino y masculino, en sociología, se utiliza para denominar al género femenino, asignado tradicional y mayoritariamente a la mujer, que define los roles, prácticas y estereotipos relacionados con la condición de mujer y puede variar en distintas culturas. Mientras que el sexo femenino se define por la producción de óvulos u otras características biológicas, el género femenino se asocia a características culturales.

En sociología, se utiliza masculino para denominar al género hegemónica y socialmente asignado a las personas de sexo biológico masculino. La persona identificada con el género masculino se llama varón aunque también se utiliza la palabra hombre en este sentido. El sexo masculino se define por la producción de espermatozoides u otras características definidas.

En la veracidad de los eventos, es aportar valor, es decir, tiene que ser útil para que la organización y quien tome de decisiones. Se obtienen mejores resultados con base en los datos que analicen. Los datos, de cualquier ámbito, no tienen utilidad si no son veraces. Con el propósito de reunir información con un conjunto de características específicas para que pueda ser valiosa.

Primero, es necesario entender que la utilidad, siendo una característica fundamental que debe aportar la información, es la cualidad de adecuarse a las necesidades de la investigación. Por consiguiente, las características que deben de cumplir los datos para que sean útiles so n: confiabilidad, relevancia, comprensibilidad.

La veracidad de los datos es el punto clave. Considera la comparabilidad, relevancia y confiabilidad. Cuyo propósito es que la información sea útil para considerar que sea verdad. Los estudiantes manifiestan que el Google Classroom es una herramienta de gran ayuda. Permite afianzar conocimientos, interactuar con el docente y estudiantes, compartir archivos. Para contribuir en el proceso de aprendizaje, y además los estudiantes consideran que todas las personas, deben adaptarse a los cambios y buscar soluciones en diversas situaciones que se puedan presentar.

En la objetividad y solidaridad, expresa la realidad tal cual es, apoyando una causa o al interés de otros. Consiste en enunciados en tiempos y modos verbales, persona verbal sin excluir a nadie, disponibilidad en escuchar y celebrar hechos de vida. Para logar una actitud real de unidad basada en metas o interés comunes. (Viñuales, 2015)

Google Classroom es una red social educativa. Basado en el intercambio de documentos, donde el estudiante puede ver todas las tareas en una página específica, el docente puede enviar notificaciones al alumnado para iniciar debates, los alumnos pueden compartir recursos con sus compañeros y ofrecer respuestas a preguntas en el muro de novedades. Por lo tanto, permite un aprendizaje colaborativo. 
Para la producción circulación y recepción, el video testimonial se difundió a través de las redes sociales, que son páginas que permiten a las personas conectarse con sus amigos e incluso realizar nuevas amistades. Con la finalidad de compartir contenidos, interactuar y crear comunidades sobre intereses similares: trabajo, lecturas y juegos.

De la apreciación de las realidades, es la observación que se hace. Consiste en rechazar, modificar o mejorar una idea o una propuesta. Contribuyendo en la evaluación de la ocurrencia de los hechos ocurridos

Los usuarios, dieron sus puntos de vista mediante un like y comentarios positivos para el uso de Google Classroom en el logro de desarrollo de proyectos de emprendimiento. Siendo un aula de clases virtual en la educación a distancia compartiendo archivos, videos, evaluaciones y otros entre docentes y estudiantes. Cuya finalidad es continuar con la educación en estos tiempos de pandemia.

El MINEDU establece el uso de recursos tecnológicos para el desarrollo de Aprendo en Casa, el docente asumió el reto de aplicar Google Classroom para el logro de desarrollo en la gestión de proyectos de emprendimiento. Considerando las necesidades y/o problemas económicos o sociales de la realidad de los estudiantes.

En la descripción del testimonio, hace referencia a un discurso en primera persona en el que se relatan las experiencias de un individuo sobre diversos acontecimientos. Está vinculado a una afirmación de una demostración o evidencia de la veracidad de una cosa. Para dar a conocer diversas experiencias de hechos reales.

Los estudiantes manifestaron el siguiente testimonio:

Se organizó los cursos asignados de cada día, semana. Los docentes pueden brindar los trabajos y notas a los estudiantes como también enviamos evidencias fotos y videos. Permite ver la fecha límite de las tareas, hasta cuando debemos entregar, es una herramienta de gran ayuda, para afianzar conocimientos, interactuar con el docente y estudiantes, compartir archivos, ayuda mutua.

Google Classroom es de gran ayuda en este tiempo al publicar sus tareas todos los inscritos pueden acceder a estas para así poder mejorar y ayudarse entre ellos, ningún participante se retrasará.

Adaptarse a los cambios es parte de ser un buen estudiante, buscar soluciones a diversas situaciones que se puedan presentar. Al comienzo de clases presenciales a virtuales no fue tan sencillo, todo era nuevo uno se adapta a la nueva forma para realizar lo que quiere realizar, en la vida siempre va existir cambios. Un estudiante debe estar preparado para afrontar cualquier situación.

Al principio trabajaba en forma individual, no le gustaba trabajar con los demás luego pensó que la unión hace la fuerza al hacer diapositivas en equipo se pudo presentar un buen trabajo.

El trabajo en equipo nos permite captar mayor cantidad de ideas para el trabajo, lo enriquece. Permite ayudarse, respetarse.

Si soy creativa al tener imaginación, uno es creativo, nosotros somos conscientes cuando llega una idea perfeccionamos lo que vemos a nuestro alrededor. Muy pronto vamos abandonar nuestro hogar estaremos sacrificando nuestra comodidad, hay que tener cuenta que, si queremos ser mas en el futuro, es necesario tomar riesgos, ser ejemplo para los demás.

El que no arriesga no gana, tenemos que tomar riesgos, puede surgir complicaciones para ello superar las dificultades, todo va valer la pena para conseguir lo que deseamos.

El objetivo que nos trazamos vamos a conseguirlo, todo está nosotros mismos, los demás no lo van hacer, tú mismo tienes que sacrificarte para lograr el objetivo.

En la interpretación de la verdad emergente, es atribuir a algo un significado determinado, adecuación entre una proposición y el estado de cosas que expresa. Consiste en que la verdad tiene una existencia objetiva, independiente de que la conozcamos o no, esta relación existe o no existe, no admite grados: si la verdad es la relación entre el juicio y la situación objetiva. Para lograr una verdad satisfactoria de la narrativa testimonial. 
La aplicación de Google Classroom influye de manera satisfactoria en el logro del desarrollo de la gestión de proyectos de emprendimiento de los estudiantes en esta educación secundaria en estos tiempos de pandemia. Que permite compartir información directamente, experimentar un ambiente educativo diferente al tradicional y por ser una aplicación ágil y organizada. Tiene por finalidad generar alternativas de solución de acuerdo a las necesidades encontradas en su entorno de carácter económico o social.

\section{Conclusiones}

El uso de Google Classroom testimonial es de gran ayuda para el desarrollo de gestión de proyectos de emprendimiento, por la interacción entre docentes y estudiantes de manera participativa permitiendo un trabajo colaborativo.

Facilita a los estudiantes en el desarrollo de aprendizajes en los aspectos relacionados en lo intelectual, personal, inter e intrapersonal y en el emprendimiento. Logrando así, una mentalidad emprendedora: con creatividad, innovación, liderazgo, trabajo en equipo, toma de decisiones, resolución de problemas. Encaminado hacia una formación integral y formativa. 


\section{Referencias Bibliográficas}

Cerrón Rojas, W. (junio de 2019). La investigación cualitativa en la educación. Horizonte de la ciencia, 163.

Manual-Google-Classroom. (s.f.). Obtenido de Manual-Google-Classroom: https://distancia.cuaed.unam.mx/ descargas/Manual-Google-Classroom.pdf

MINEDU. (2016). Curricula nacional de educación básica. Lima.

Rodriguez, Y. (2019). Aprendizaje colaborativo en entornos virtuales. Maestría en Innovación en educación, Universidad Andina Simón Bolivar.

Velez, M. (2016). Google Classroom en la enseñanza: Manual sobre funciones básicas y mejores prácticas de uso. Centro. Puerto Rico: Centro para la excelencia académica.

Viñuales, A. (16 de 02 de 2015). antonioviñuelas.com. Obtenido de http://www.xn--antonioviualesynb. com/2015/02/16/caracteristicas-de-la-objetividad-y-la-subjetividad/\# 